\title{
Investigation of measuring multiple biological signals by analog circuit dynamic reconfiguration
}

\author{
Tetsuya Suematsu, Yuta Suzuki, Akira Yamawaki \\ Kyushu Institute of Technology, Kitakyushu, 804-8550 Japan \\ *Corresponding Author: yama@ecs.kyutech.ac.jp
}

\begin{abstract}
For high-mix low-volume electronic products, we have to prepare the dedicated manufacturing line for each product. As the life cycle of electronic products becomes shorter and shorter, we have also to change the manufacturing line corresponding to the new product. These cost cannot be neglected to gain enough income. So, a uniformed device platform that can be used for many kinds of product is desired. The reconfigurable device supporting analog circuit reconfiguration is one of the dedicated device platform.

However, dynamic reconfiguration of analog circuits has not been widely used. This is because how to use the analog circuit dynamic reconfiguration for many kinds of application is not well known and its design environment has not been unified in comparison with digital circuits.

To tackle this problem, we think that many case studies of application using analog circuit dynamic reconfiguration are significant. This paper attempts to use it for the health care field. Concretely, single device measures multiple biological signals by dynamically changing the tailored analog circuits.

At first, we investigate how to measure the heart rate by photo reflector on the commercial device supporting analog circuit dynamic reconfiguration, PSoC. Next, we investigate how to measure the myoelectric potential by electrode pads on PSoC. The experimental results show that the PSoC can acquire the desired waveform of the heart rate and the myoelectric potential. This fact indicates that single device supporting the analog circuit dynamic reconfiguration can provide the unified single device over the several kinds of biological signals.
\end{abstract}

Keywords: heart rate, myoelectric potential, PSoC.

\section{Introduction}

For high-mix low-volume electronic products, we have to prepare the dedicated manufacturing line for each product. As the life cycle of electronic products becomes shorter and shorter, we have also to change the manufacturing line corresponding to the new product. These costs cannot be neglected to gain enough income. So, a generic device platform that can be manufactured with single product line that can be applied to many kinds of electronic products uniformly is desired.

To realize such uniformed device platform, we think circuit dynamic reconfiguration is useful. For the dynamic circuit reconfiguration, the digital circuit has been much focused on and the technology has been widely used. But the study of analog circuit dynamic reconfiguration has been fewer taken attention, and the technology has been not widely used. This is because for two reasons.

First, the applications of analog circuit dynamic reconfiguration have not been investigated. So, we don't know well how to use it efficiently to realize stable products compared with digital circuits. Second, this is because its design environment hasn't been unified to support dynamic reconfiguration.

We have attempted to accomplish a concrete generic way to use analog circuit dynamic reconfiguration through many case studies. This research concentrates to the application of health awareness. We show the way to measure the heart rate by photo reflector and the myoelectric potential by electrode pads to use the analog circuit reconfiguration on PSoC. 


\section{Measurement Method}

As the device supporting analog circuit dynamic reconfiguration, we employ $\mathrm{PSoC}$ microcomputer in this paper.

\subsection{Heart Rate Measurement}

First, the heart rate means the same as the pulsation The heart rate's measurements have several ways. These are electrocardiograph and blood pressure meter method, heart sound projection, photoelectric pulse wave method Electrocardiograph is the way measures the electric pulse arising by the cardiac cycle in the body. Blood pressure meter method is the way measures the blood vessel pressure's change along heartbeat. Heart sound is the way that measures the sound arising along the heartbeat.

And photoelectric pulse wave method is the heart rate's measurement. This method of measurement is transmission type pulse wave measurement and reflected pulse wave measurement. The reflected pulse wave measurement is the method that measures the blood flow's variation that irradiates the body surface with the infrared rays or the red light and changes along the heartbeat as the variation of light permeating within the body.

In this research, we employ the reflected pulse wave measurement to obtain the heart rate. This method irradiates the body with the infrared rays or the red light and so on Then, it measures the internal reflected light by using photo-diode or photo-transistor.

The method measures the pulsation signal by measuring the blood changing along the heartbeat because oxygenated hemoglobin exists in the blood of the artery and absorbs incident light. This method is independent on the measurement location.

\subsection{Proposed Measurement of Heart Rate}

The sensor using in this research is a photo-reflector. This sensor means infrared ray LED and photo-transistor. The measurement puts finger on the sensor. Fig.1 shows the circuit to measure the heart rate by using PSoC.

The user puts the finger on the photo-reflector. The photo-transistor in the photo-reflector receives the light reflected from the finger by infrared rays LED.

The ambient light in the photo-transistor's output is removed by some filter. The filtered output is input to PSoC. The amplifier in the PSoC amplifies the filtered wave by sixteen times.

The heart rate's frequency is from $0.3 \mathrm{~Hz}$ to $3 \mathrm{~Hz}$. The

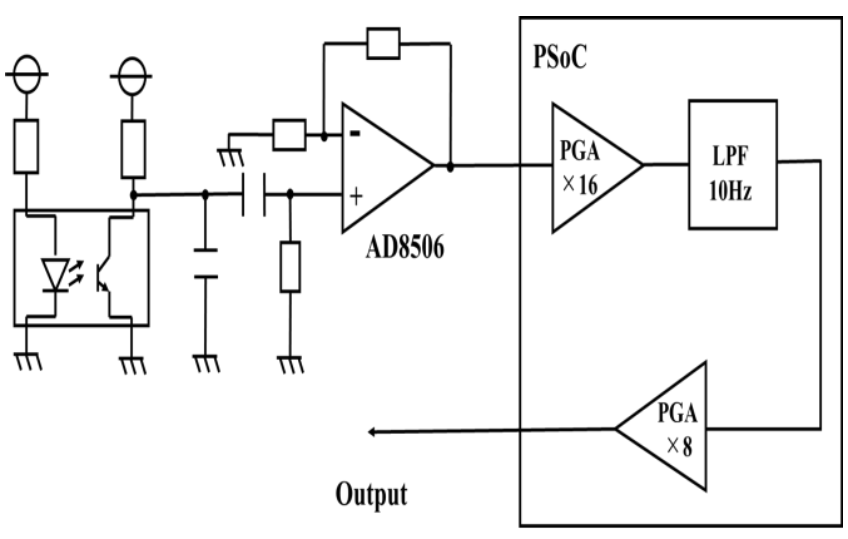

Fig.1. Circuit Diagram to Measure Heart Rate

amplified input is passed through a low-pass filter of $10 \mathrm{~Hz}$ to remove the noise of the signal. Finally, the filtered wave is amplified by eight times and the gained output is observed as the measured heart rate.

\subsection{Myoelectric Potential Measurement}

The measurements of myoelectric potential have three ways. These are the measurement by needle electrodes, the measurement by surface electrodes, the measurement by wire electrodes.

The measurement by the needle electrodes inserts the thin needle into the subject's arm, and measures the action electric potential.

The measurement by the surface electrodes measures the action electric potential of volumetric conduction on the subject's skin. And the surface electrodes are put on the subject's arm.

The measurement by wire electrodes inserts wire electrodes into the subject's arm by using a syringe, measures the action electric potential after removing the syringe.

In three measurements, the needle electromyogram leads the local electromyogram to examine the nerve damage and the pathology of the muscle. So, the user shifts the needle electrodes, evaluates whether the subject has abnormal finding of various muscle.

On the other hand, the measurement by the surface electrodes uses evoked electromyogram that uses electric stimulation to induce action electric potential, often uses operation analysis because it evaluates an action of a muscle fiber in the field of rehabilitation.

And it uses biofeedback therapy that control muscle activity.

We employ the measurement by the surface electrodes in this research. 


\subsection{Proposed Measurement of Myoelectric Potential}

In this research, the sensors use the electrode pads. Fig.2. shows the state of the subject's arm sticking the pads. We stick the pads on the protractor muscle of arm. This is because it is comparatively to measure the myoelectric potential of the muscle ${ }^{(1)}$.

The measurement is that these sensors are put on the subject's arm, measure the potential got from the sensors. Fig. 3 shows the heart rate measurement circuit on PSoC.

First, the signal from the electrode pads is amplified by instrumentation amplifier with one thousand times gain. This is because the signal from the electrode pads is too small, PSoC doesn't treat the signal.

The amplified signal is sent to PSoC. And the input signal passes through amplifier of two times, removes the noise through the low-pass filter of $500 \mathrm{~Hz}$.

The signal is measured in this research. We compare the result with the output of the commercially available measurement circuit's output. By doing it, we know whether the created circuit's output is correct or wrong.

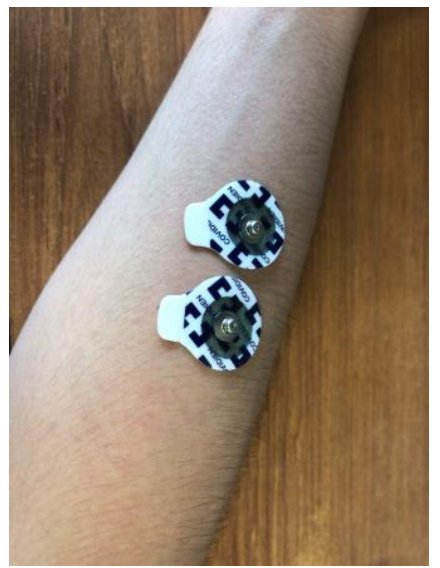

Fig.2. Arm with Pads Stickled

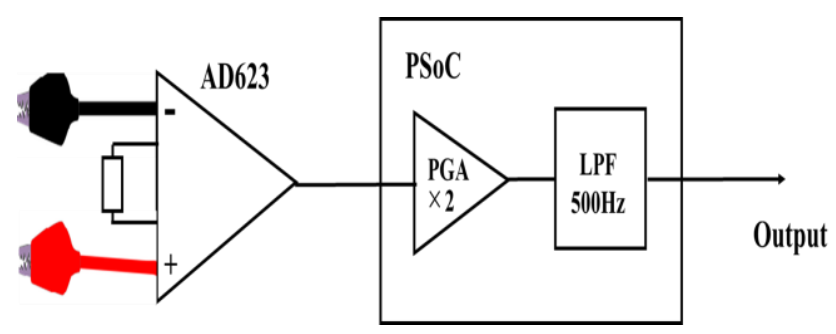

Fig.3.Circuit to Measure Myoelectric Potential

\section{Experiment}

\subsection{Result of Heart Rate}

We measured the heart rate by the circuit of Fig.1. The subject is a 24 years old man. Fig.4. shows the output of PSoC. And the heart rate of Fig.4. is sixty-three. The general heart rate is from sixty to eighty.

Compared with its number, the result's number is within the limits of its number. So, the heart rate measure is collect.

\subsection{Result of Myoelectric Potential}

We measured the myoelectric potential by the circuit of Fig.3. The subject is a 24 years old man.

Fig.5. shows the output of PSoC. Fig.5. shows that the circuit' s output is big when the subject puts some muscle and its output is certain when he doesn't put some muscle. And the commercially available measurement circuit's output shows Fig.6. These graph similarly shows that the circuit's output is big when the subject puts some muscle and its output is certain when he doesn't put some muscle. Compared with the commercial circuit and the fabricated circuit, the myoelectric potential measurement is correct because these output is same signal.

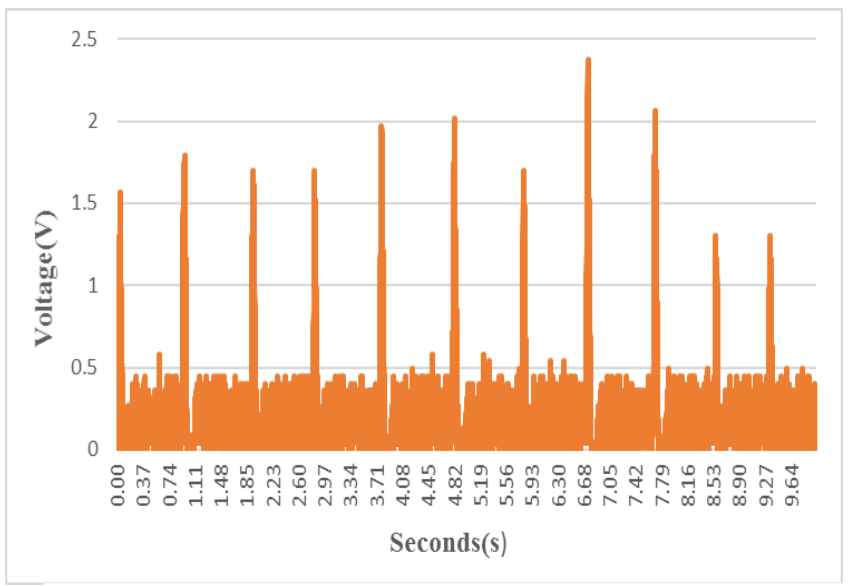

Fig.4. Result of Heart Rate

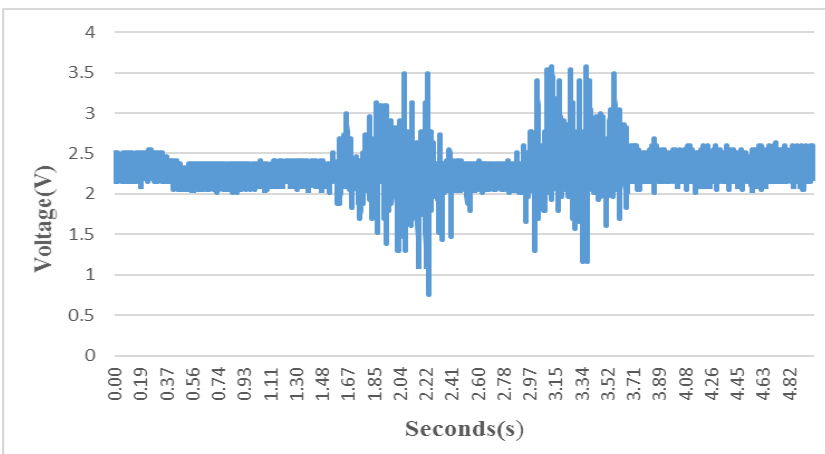

Fig.5. Result of Myoelectric Potential 


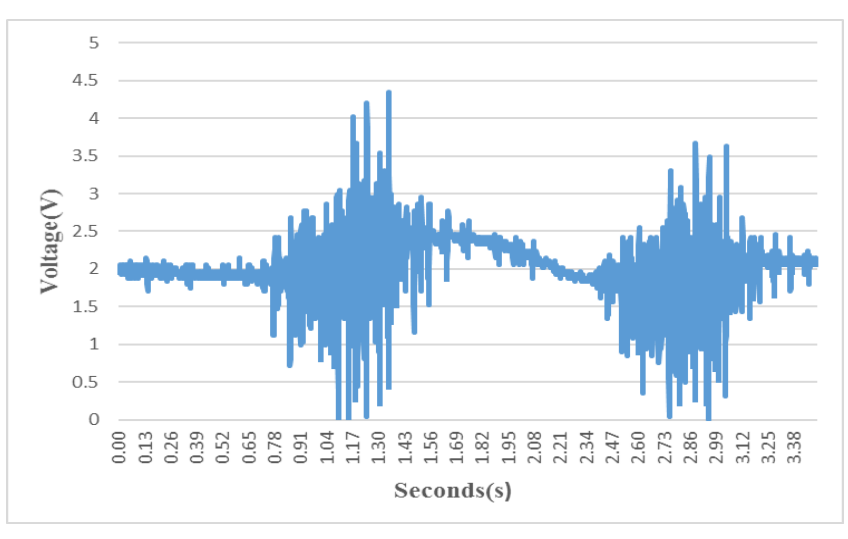

Fig.6. Commercial Circuit's Output

\subsection{Consideration}

We confirm that the two biological signal can be measured by PSoC.

Fig.7. shows the circuits doing analog circuit dynamic reconfiguration. Two external measurement circuit's outputs is sent to each pin of PSoC. And PSoC dynamically changes the wiring to each pin and the desired circuit structure. By doing it, we think one device measures two biological by using analog circuit dynamic reconfiguration.

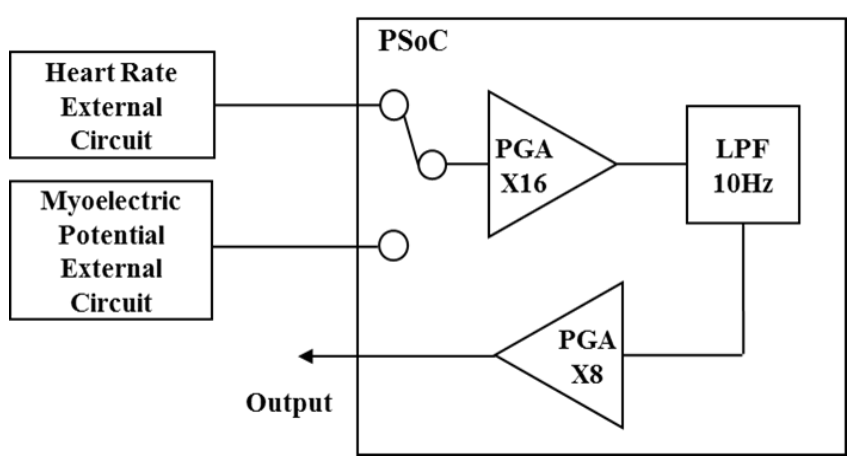

(a) Circuit Diagram to measure Heart Rate

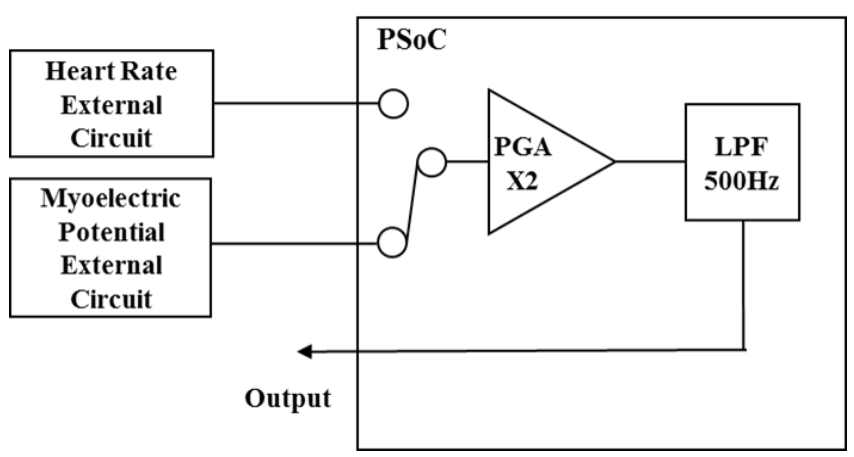

(b) Circuit to measure myoelectric potential

Fig.7. Circuits Diagram to do reconfiguration

\section{Conclusion}

This paper has proposed the method to measure the heart rate and the myoelectric potential on PSoC. Each measurement method confirms that it measures the two biological signal without problem.

As future work, we employ the measurement methods, plan to measure the two biological signals by using analog circuit dynamic reconfiguration. And, for general use, we will develop the system that the data of the measurement result is sent to $\mathrm{PC}$ or the smartphone.

\section{References}

(1) Tomohiro Kizuka, Tadashi Masuda, Toru Sadoyama: "Surface Electromyogram", 2006 in Japanese

(2) Teiji Kimura: "Muscle Strength and Electro myography", Exercise physiology for physical therapy, Vol. 7, No. 3, pp. 175-187, 1992 